\title{
Trigeminal Medullary Dorsal Horn Neurons Activated by Nasal Stimulation Coexpress AMPA, NMDA, and NK1 Receptors
}

\author{
P. F. McCulloch, K. M. DiNovo, D. J. Westerhaus, T. A. Vizinas, \\ J. F. Peevey, M. A. Lach, and P. Czarnocki \\ Department of Physiology, Chicago College of Osteopathic Medicine, Midwestern University, 555 31st Street, \\ Downers Grove, IL 60515, USA \\ Correspondence should be addressed to P. F. McCulloch; pmccul@midwestern.edu
}

Received 16 August 2013; Accepted 7 October 2013

Academic Editors: A. Almeida and M. Chacur

Copyright (C) 2013 P. F. McCulloch et al. This is an open access article distributed under the Creative Commons Attribution License, which permits unrestricted use, distribution, and reproduction in any medium, provided the original work is properly cited.

\begin{abstract}
Afferent information initiating the cardiorespiratory responses during nasal stimulation projects from the nasal passages to neurons within the trigeminal medullary dorsal horn $(\mathrm{MDH})$ via the anterior ethmoidal nerve (AEN). Central AEN terminals are thought to release glutamate to activate the MDH neurons. This study was designed to determine which neurotransmitter receptors (AMPA, kainate, or NMDA glutamate receptor subtypes or the Substance P receptor NK1) are expressed by these activated MDH neurons. Fos was used as a neuronal marker of activated neurons, and immunohistochemistry combined with epifluorescent microscopy was used to determine which neurotransmitter receptor subunits were coexpressed by activated MDH neurons. Results indicate that, during nasal stimulation with ammonia vapors in urethane-anesthetized Sprague-Dawley rats, activated neurons within the superficial MDH coexpress the AMPA glutamate receptor subunits GluA1 (95.8\%) and GluA2/3 (88.2\%), the NMDA glutamate receptor subunits GluN1 (89.1\%) and GluN2A (41.4\%), and NK1 receptors (64.0\%). It is therefore likely that during nasal stimulation the central terminals of the AEN release glutamate and substance $\mathrm{P}$ that then produces activation of these MDH neurons. The involvement of AMPA and NMDA receptors may mediate fast and slow neurotransmission, respectively, while NK1 receptor involvement may indicate activation of a nociceptive pathway.
\end{abstract}

\section{Introduction}

The diving response, an autonomic reflex characterized by apnea, bradycardia, and increased peripheral vascular resistance, is initiated through nasal stimulation or when animals submerge under water [1]. Afferent information involved in initiating this response projects from the nasal mucosa to the spinal trigeminal nucleus via the anterior ethmoidal nerve (AEN), a branch of the ophthalmic division of the trigeminal nerve [2]. The central projections of the AEN terminate within the spinal trigeminal nucleus caudalis [3]. This area is also known as the medullary dorsal horn (MDH) due to its functional and anatomical similarities with the spinal dorsal horn (SDH) [4]. Second-order neurons within the $\mathrm{MDH}$ are activated both during voluntary diving in conscious animals [5] and nasal stimulation in anesthetized animals [6-8]. It is likely that excitatory amino acids serve as neurotransmitters at synapses within the $\mathrm{MDH}$ that are part of this response, as injection of kynurenate, a general excitatory amino acid antagonist, into the MDH abolishes the cardiorespiratory responses to nasal stimulation [9]. More specifically, it is likely that glutamatergic neurotransmission in the $\mathrm{MDH}$ after nasal stimulation is at least partially dependent on the NMDA subtype of glutamate receptors [8]. However, other glutamate receptor subtypes and other neurotransmitters/neuromodulators may also be involved in the neurotransmission between the AEN and MDH secondary neurons.

Glutamate is the major excitatory neurotransmitter in the central nervous system [10-12]. It activates both ligand-gated ionotropic glutamate receptors and G-protein-coupled metabotropic glutamate receptors $[10,12]$. Based on their agonist specificities, ionotropic receptors, those receptors that are directly coupled to cation channels, are divided 
into $\alpha$-amino-3-hydroxy-5-methyl-4-isoxazole-propionate (AMPA), kainate, and N-methyl-D-aspartate (NMDA) receptors $[10,12,13]$. Ionotropic glutamate receptors are formed from heteromeric subunit assemblies that have different physiological and pharmacological properties [12]. The subunit composition determines the biophysical properties of the receptor, and the subunits that comprise these are specific for each of the three ionotropic glutamate receptor families $[11,13]$. Ionotropic glutamate receptors are formed from tetrameric assemblies of different subunits [12], and the four subunits are arranged with three transmembrane-spanning and one pore-lining domain $[12,14]$.

AMPA and kainate receptors collectively are known as non-NMDA receptors $[10,13]$. AMPA receptors mediate fast excitatory neurotransmission in most synapses within the CNS $[10,12,14]$ and are typically concentrated at the postsynaptic sites of excitatory synapses [15]. Subunits that compose AMPA receptors include GluA1, GluA2, GluA3, and GluA4 $[10,12,14]$. Kainate receptors exhibit fast activation similar to AMPA receptors but are thought to fulfill more of a neuromodulatory role in the CNS [16]. Five kainate receptor subunits have been identified: GluK1, GluK2, GluK3, GluK4, and GluK5 $[10,14]$. Kainate and AMPA receptors subunits can coexist in the same neurons but do not appear to coassemble with each other [10]. At most central synapses, both AMPA and NMDA receptors are activated during synaptic transmission. AMPA receptors mediate fast neurotransmission while neurotransmission mediated by NMDA receptors occurs more slowly and lasts much longer $[10,13]$. The fundamental NMDA receptor subunit is GluN1, with modulatory subunits being GluN2A, GluN2B, GluN2C, GluN2D, and GluN3 [10, $12,14]$.

Neuropeptides and kinins are important messengers in the nervous system [17]. The neurokinin substance P (SP), an 11-amino acid peptide [18-20], was identified as the first member of the tachykinin family [19-21]. The neurokinin receptor $\mathrm{NK1}$ is preferentially activated by SP and is a G-protein-coupled receptor with seven transmembranespanning domains $[18,22]$. SP, acting at NK1 receptors, elicits excitatory effects as a neurotransmitter or neuromodulator in both the central and peripheral nervous systems [17, 18], although the functional role of tachykinins could be related to an interaction with glutamate acting at NMDA receptors at the postsynaptic site $[23,24]$. SP and NK1 receptors function in slow nociceptive neurotransmission, primarily in tissue injury and inflammation rather than in acute pain $[17,21,24]$.

The present study was designed to characterize the $\mathrm{MDH}$ neurons involved in mediating the cardiorespiratory changes induced after nasal stimulation by determining the types of neurotransmitter receptors that are expressed by these activated MDH neurons. Fos was used as a neuronal marker of activated neurons [25], and immunohistochemistry was used to determine which neurotransmitter receptor subunits were coexpressed by activated $\mathrm{MDH}$ neurons. The identification of which type of ionotropic glutamate receptor (AMPA, kainite, or NMDA) and whether SP is involved would help describe the specifics of the afferent signal and the possible integration of that signal within second-order MDH neurons.

\section{Materials and Methods}

All experimental procedures were approved by the Midwestern University IACUC. Male Sprague-Dawley rats (258$563 \mathrm{~g} ; N=37$ ) were obtained from a commercial vendor (Harlan). Brain tissue from some animals was used to test more than one Fos/neurotransmitter receptor combination (see below).

2.1. Stimulation of Nasal Passages. The nasal passages of rats were stimulated with ammonia vapors to activate $\mathrm{MDH}$ neurons. To reduce animal usage, control experiments using unstimulated animals were not repeated here, as unstimulated animals show neither cardiorespiratory changes nor activation of $\mathrm{MDH}$ neurons [6,7]. For complete experimental details, see Rybka and McCulloch [6]. Briefly, rats were initially anesthetized with 5\% isoflurane (in 95\% $\mathrm{O}_{2} / 5 \%$ $\mathrm{CO}_{2}$ ) and then transferred to a nose cone delivering $2-3 \%$ isoflurane. The right femoral artery and vein were cannulated to record blood pressure and administer drugs, respectively. The trachea was cannulated caudally to enable ventilation, and rostrally to aid in stimulation of the nasal passages. Respiratory rate was monitored through thermal sensing of air flow in the caudal tracheal cannula. After surgery was complete, isoflurane was withdrawn as urethane $(1300 \mathrm{mg} / \mathrm{kg}$, iv) was slowly injected. Rats rested for approximately $1 \mathrm{hr}$ to achieve a stable plain of urethane anesthesia before the start of experiments. Body temperature was maintained at $37 \pm 1^{\circ} \mathrm{C}$. Electronic signals for respiration and arterial blood pressure $(\mathrm{BPa})$ were recorded, stored and analyzed using appropriate computer software (Spike2, CED). Heart rate (HR) was determined from pulse pressure intervals.

A stimulation trial consisted of placing a cotton swab soaked in ammonia $2-3 \mathrm{~mm}$ in front of the external nares for $5 \mathrm{~s}$. A suction pump connected to the rostral tracheal cannula gently drew ammonia vapors through the nasal passages. Stimulations occurred every $5 \mathrm{~min}$ for $2 \mathrm{hr}$ for a total of 24 trials. A $1 \mathrm{hr}$ wait followed the final stimulation to allow activated neurons to produce Fos. At the end of experiments rats were euthanized with concentrated sodium pentobarbital (0.3 mL Sleepaway, iv; Fort Dodge), followed by a transcardiac perfusion with a $300 \mathrm{~mL}$ phosphate buffered saline (PBS) solution containing $0.25 \%$ procaine and then $300 \mathrm{~mL}$ $4 \%$ paraformaldehyde. Finally, the brains were removed and stored in a PBS solution containing 4\% paraformaldehyde and $20 \%$ sucrose. The brains postfixed for a minimum of $24 \mathrm{hr}$ at $4^{\circ} \mathrm{C}$, and the brainstems were then cut transversely at 40 or $50 \mu \mathrm{m}$ using a freezing microtome.

2.2. Immunohistochemistry. PBS was used for all immunohistochemical washes. Free floating (1:3 series) brainstem sections were blocked in 10\% normal donkey serum for $1 \mathrm{hr}$. The sections were incubated overnight with an anti-Fos primary antibody (see Table 1). The next day the sections were blocked for 1 hour in $10 \%$ bovine serum albumin (BSA). Next, the sections were incubated with a fluorescent-tagged secondary antibody directed against the Fos primary antibody for $2 \mathrm{hr}$. The sections were then blocked with $10 \%$ normal 
TABLE 1: Primary and secondary antibody combinations. Based on availability of primary and secondary antibodies and results obtained in preliminary experiments, antibodies, fluorescent color combinations, and dilutions were changed as necessary. For catalog product numbers, C: Chemicon; I: Invitrogen; M: Millipore; MP: Molecular Probes; and SC: Santa Cruz. For fluorescent tagged secondary antibodies, AF: AlexaFluor.

\begin{tabular}{|c|c|c|}
\hline \multicolumn{3}{|l|}{ AMPA } \\
\hline \multicolumn{3}{|c|}{ GluA1 (GluR1) and Fos $(N=6)$} \\
\hline & Fos (red) & GluA1 receptor subunit (green) \\
\hline Primary Ab: & goat (SC, sc-52G); 1:1000 & rabbit $(\mathrm{M}, \mathrm{AB} 1504) ; 1: 10,000$ \\
\hline Secondary Ab: & donkey (MP, A11058; AF594); 1:500 & donkey (MP, A21206; AF488); 1:500 \\
\hline \multicolumn{3}{|c|}{ GluA2/3 (GluR2/3) and Fos $(N=4)$} \\
\hline & Fos (green) & GluA2/3 receptor subunits (red) \\
\hline Primary Ab: & goat (SC, sc-52G); $1: 2500$ & rabbit $(\mathrm{C}, \mathrm{AB} 1506) ; 1: 2500$ \\
\hline Secondary Ab: & donkey (MP, A11055; AF488); 1:1000 & donkey (MP, A21207; AF594); 1:1000 \\
\hline \multicolumn{3}{|c|}{ GluA (GluR4) and Fos $(N=3)$} \\
\hline & Fos (red) & GluA4 receptor subunit (green) \\
\hline Primary Ab: & goat (SC, sc-52G); $1: 1000$ & rabbit (M, AB1508); 1:5000 \\
\hline Secondary Ab: & donkey (MP, A11058; AF594); 1:500 & donkey (MP, A21206; AF488); 1:500 \\
\hline \multicolumn{3}{|l|}{ Kainate } \\
\hline \multicolumn{3}{|c|}{ GluK1/2/3 (GluR5/6/7) and Fos $(N=3)$} \\
\hline & Fos (green) & GluK1/2/3 receptor subunits (red) \\
\hline Primary Ab: & rabbit (SC, sc-52); $1: 1000$ & mouse (M, MAB379); 1:5,000 \\
\hline Secondary Ab: & donkey (MP, A21206; AF488); 1:500 & donkey (MP, A21203; AF594); 1:500 \\
\hline \multicolumn{3}{|c|}{ GluK4 (KA1) and Fos $(N=6)$} \\
\hline & Fos (green) & GluK4 receptor subunit (red) \\
\hline Primary Ab: & goat (SC, sc-52G); $1: 2000$ & rabbit (SC, sc-25700); 1:100 \\
\hline Secondary Ab: & donkey (I, A11055; AF488); 1:500 & donkey (I, A21207; AF594); 1:1000 \\
\hline \multicolumn{3}{|c|}{ GluK5 (KA2) and Fos $(N=5)$} \\
\hline & Fos (green) & GluK5 receptor subunit (red) \\
\hline Primary Ab: & goat (SC, sc-52G); 1:2000 & rabbit (SC, sc-25701); 1:200 \\
\hline Secondary Ab: & donkey (I, A11055; AF488); 1: 1000 & donkey (I, A21207; AF594); 1: 1000 \\
\hline \multicolumn{3}{|c|}{ NMDA } \\
\hline \multicolumn{3}{|c|}{ GluN1 (NR1, NMDA $\zeta 1)$ and Fos $(N=5)$} \\
\hline & Fos (green) & GluN1 receptor subunit (red) \\
\hline Primary Ab: & rabbit (SC, sc-52); 1:5000 & goat (SC, sc-1467); $1: 700$ \\
\hline Secondary Ab: & donkey (I, A21206; AF488); 1: 1000 & donkey (I, A11058; AF594); 1: 1500 \\
\hline \multicolumn{3}{|c|}{ GluN2A (NR2a, NMDAc1) and Fos $(N=6)$} \\
\hline & Fos (green) & GluN2A receptor subunit (red) \\
\hline Primary Ab: & goat (SC, sc-52G); 1:2000 & rabbit (SC, sc-9056); 1:200 \\
\hline Secondary Ab: & donkey (I, A11055; AF488); $1: 1000$ & donkey (I, A21207; AF594); 1:1000 \\
\hline \multicolumn{3}{|c|}{ GluN2B (NR2b, NMDAc2) and Fos $(N=6)$} \\
\hline & Fos (green) & GluN2B receptor subunit (red) \\
\hline Primary Ab: & goat (SC, sc-52G); $1: 2000$ & rabbit (SC, sc-9057); 1:500 \\
\hline Secondary Ab: & donkey (I, A11055; AF488); 1: 1000 & donkey (I, A21207; AF594); 1:1000 \\
\hline \multicolumn{3}{|c|}{ GluN2C (NR2c, NMDAc3) and Fos $(N=3)$} \\
\hline & Fos (green) & GluN2C receptor subunit (red) \\
\hline Primary Ab: & goat (SC, sc-52G); $1: 1000$ & rabbit (SC, sc-50437); 1:200 \\
\hline Secondary Ab: & donkey (I, A11055; AF488); 1: 1000 & donkey (I, A21207; AF594); 1: 750 \\
\hline \multicolumn{3}{|c|}{ GluN2D (NR2d, NMDA\&4) and Fos $(N=3)$} \\
\hline & Fos (green) & GluN2D receptor subunit (red) \\
\hline Primary Ab: & goat (SC, sc-52G); $1: 500$ & rabbit (SC, sc-10727); $1: 100$ \\
\hline Secondary Ab: & donkey (I, A11055; AF488); $1: 1000$ & donkey (I, A21207; AF594); 1:1000 \\
\hline \multicolumn{3}{|c|}{ GluN3B (NR3B) and Fos $(N=6)$} \\
\hline & Fos (green) & GluN3B receptor subunit (red) \\
\hline Primary Ab: & goat (SC, sc-52G); 1:1000 & rabbit (SC, sc-50474); $1: 150$ \\
\hline Secondary Ab: & donkey (I, A11055; AF488); 1: 1000 & donkey (I, A21207; AF594); 1:1000 \\
\hline
\end{tabular}


TABle 1: Continued.

\begin{tabular}{lcc}
\hline $\begin{array}{l}\text { Substance } \mathrm{P} \\
\mathrm{NK} 1 \text { and } \operatorname{Fos}(N=5)\end{array}$ & NK1 receptor (red) \\
& Fos (green) & guinea pig (M, AB15810); 1:2,000 \\
Primary Ab: & rabbit (SC, sc-52); 1:1000 & goat (MP, A11076; AF594); 1:500 \\
Secondary Ab: & donkey (MP, A21206; AF488); 1:500
\end{tabular}

Note. Glutamate receptor subunit names are consistent with IUBCP nomenclature, with common receptor subunit names in parentheses [14].

donkey serum for 1 hour. The sections were then incubated overnight in a second primary antibody directed against a specific neurotransmitter receptor or receptor subunit (see Table 1). The next day the sections were again blocked for $1 \mathrm{hr}$ in $10 \%$ BSA. Next, the sections were incubated with a fluorescent-tagged secondary antibody directed against the primary receptor antibody for $2 \mathrm{hr}$. Lastly, sections free floating in PBS were organized into serial order, mounted on clean slides, and coverslipped with a buffered glycerol mounting solution. All tissue processing occurred in minimal lighting in order to minimize fluorescent fading.

On some occasions tissue sections were incubated overnight with a cocktail of primary antibodies for both Fos and the specific neurotransmitter receptor (see Table 1). The following day the sections were blocked for $1 \mathrm{hr}$ in $10 \%$ BSA. Next, the sections were incubated with a fluorescenttagged secondary antibody directed against the Fos primary antibody for $2 \mathrm{hr}$. The sections were then blocked for $1 \mathrm{hr}$ in $10 \%$ BSA. Next, the sections were incubated with a fluorescent-tagged secondary antibody directed against the receptor primary antibody for $2 \mathrm{hr}$. The sections were then organized into serial order, mounted, and coverslipped.

2.3. Microscopy. A Nikon Eclipse E600 microscope with epifluorescent attachment was used to visualize the right and left $\mathrm{MDH}$ (Figure 1). An average of $20.13 \pm 1.01 \mathrm{MDH}$ hemisections (left: $10.11 \pm 0.58$; right: $9.98 \pm 0.51$ ) was used to visualize each of the 61 primary and secondary antibody pairings (see Table 1). Tissue was first inspected for the presence of Fos in the nucleus of the neuron (indicating activation of that neuron) and then for the neurotransmitter receptor or receptor subunit on the cellular membrane (indicating expression of that neurotransmitter receptor by that neuron). The number of single-labeled (Fos only) and colocalized (Fospositive and neurotransmitter receptor positive) neurons were counted bilaterally within the ventral $\mathrm{MDH}$ (both superficial (laminae I and II) and deep regions (laminae III-V)). Neurons within the ventral paratrigeminal nucleus, located adjacent to the $\mathrm{MDH}$ within the spinal trigeminal tract, were also counted. Fos-positive neurons in other brain regions were not counted. To aid in the counting and to reduce fluorescent fading of the tissue, 10x photomicrographs were taken through red and green cube filters with a digital camera (Q-Imaging) and associated imaging software (Northern Eclipse). Subsequent microscopic analysis at 20 or $40 \mathrm{x}$ and inspection of photomicrograph overlays of the red and green images verified the presence of single-labeled or colocalized neurons.

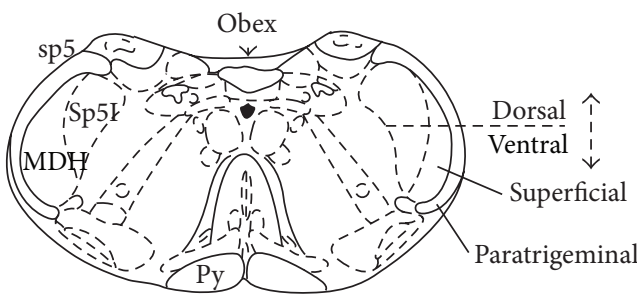

Bregma-13.68

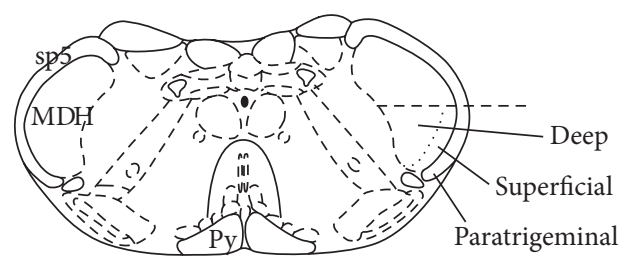

Bregma-14.08

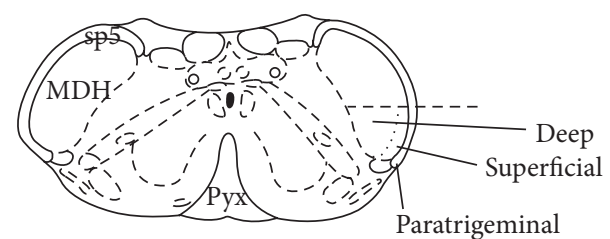

Bregma- 14.60

FIGURE 1: After stimulation of the nasal passages with ammonia vapors, Fos-positive cells were counted bilaterally in the ventral portion of the medullary dorsal horn, between the pyramidal decussation (Bregma-14.60) and obex (Bregma-13.68). Superficial is MDH laminae I\&II; Deep is MDH laminae III-V; Paratrigeminal neurons are located within spinal trigeminal tract. Cross-sections from Paxinos and Watson [26]. Abbreviations: $\mathrm{MDH}$, medullary dorsal horn; Py, pyramidal tract; Pyx, pyramidal decussation; sp5, spinal trigeminal tract, Sp5I, spinal trigeminal nucleus interpolaris.

2.4. Data Presentation. HR (bpm; beats/min), $\mathrm{BPa}(\mathrm{mm} \mathrm{Hg})$, and respiratory rate (breaths/min) are presented as mean \pm standard error (SE). Cardiovascular data were analyzed by comparing pretrial, trial, and posttrial values. Photomicrographs were adjusted using ImageJ (v1.43 g, NIH), and figures were composed and labeled using CorelDraw (Corel). Neuronal data are presented as number of Fos-positive neurons \pm SE per hemisection and as the percentage of Fos-positive neurons that coexpressed the specific neurotransmitter receptor or receptor subunit. Statistical differences were tested with Repeated Measures One-Way ANOVAs (SigmaStat, SPSS), with $P<0.05$ set as the level of significance. 


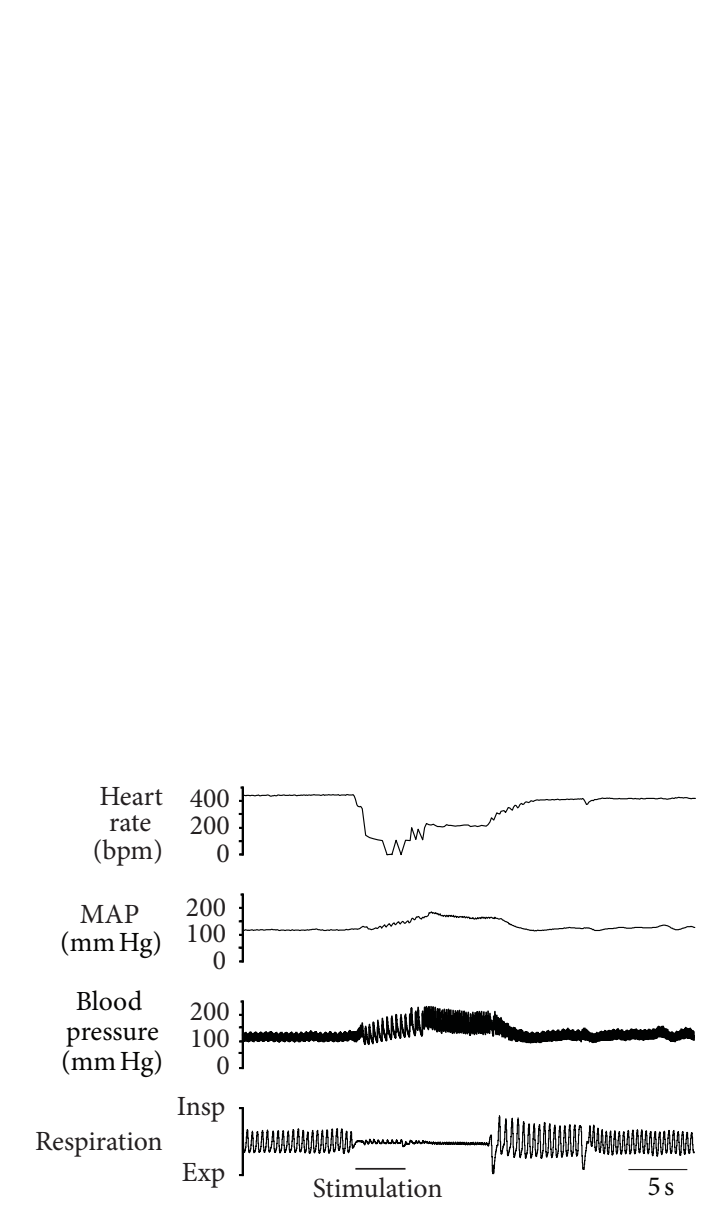

(a)
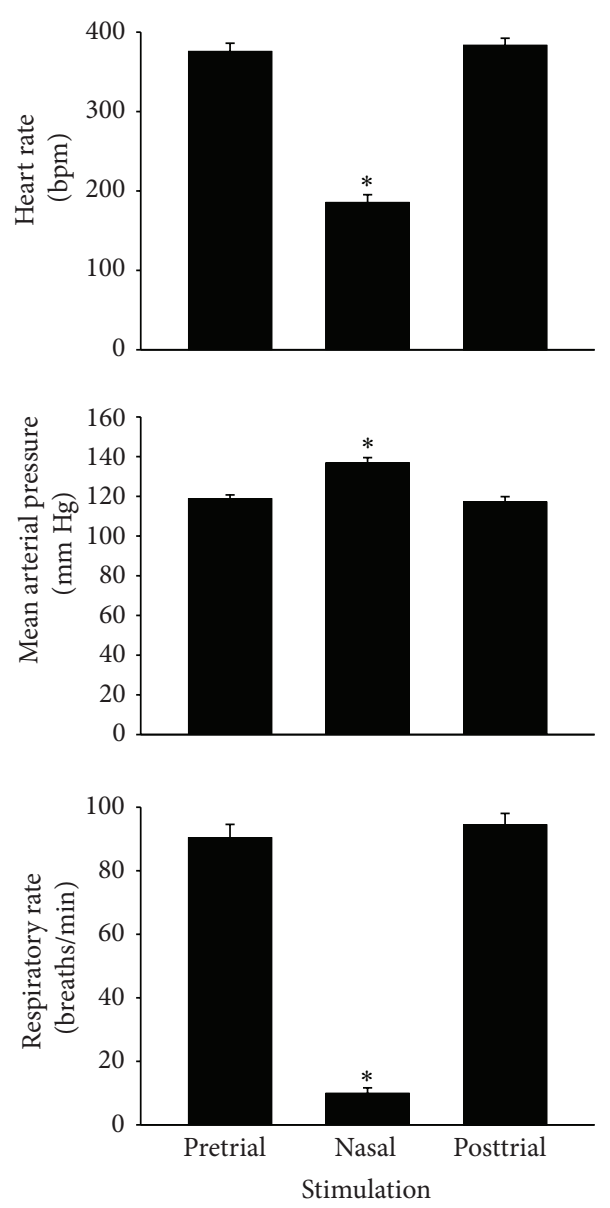

(b)

FIGURE 2: (a) Original traces showing cardiorespiratory responses to stimulation of the nasal passages with ammonia vapors (bar under trace). MAP is mean arterial pressure. (b) Heart rate, mean arterial blood pressure, and respiratory rate (mean \pm SE) before, during, and after nasal stimulation. * Significantly different from both pretrial and posttrial.

\section{Results}

Stimulation of the nasal passages with ammonia vapors produced an immediate and intense cardiorespiratory response that included bradycardia, an increase in arterial blood pressure, and apnea (Figure 2(a)). During the $5 \mathrm{~s}$ of nasal stimulation, HR decreased significantly from $375 \pm 11$ to $185 \pm 10 \mathrm{bpm}$ and mean $\mathrm{BPa}$ significantly increased from $119 \pm 2$ to $137 \pm 3 \mathrm{~mm} \mathrm{Hg}$ (Figure 2(b)). Apnea lasted an average of $10.1 \pm 0.6 \mathrm{~s}$, but if apnea was not present, respiratory rate significantly decreased from $90 \pm 4$ to $10 \pm 2$ breaths/min (Figure 2(b)).

The total Fos labeling (Fos only neurons plus Fos-positive neurons colocalized with a neurotransmitter receptor) was significantly greater in the superficial MDH, compared with the total Fos labeling in the deep $\mathrm{MDH}$ and paratrigeminal nucleus. Within each $\mathrm{MDH}$ hemisection, there were $11.74 \pm 1.07$ total Fos-positive neurons in the superficial $\mathrm{MDH}$, compared with $3.61 \pm 0.42$ and $1.43 \pm 0.18$ Fospositive neurons within the deep $\mathrm{MDH}$ and paratrigeminal nucleus, respectively. Per hemisection, $69.9 \%$ of the activated neurons were located in the superficial MDH, with $21.5 \%$ and $8.5 \%$ located in the deep $\mathrm{MDH}$ and paratrigeminal nucleus, respectively.

The number of Fos only and colocalized Fos-positive neurons (and therefore the percent of colocalized neurons) depended upon which neurotransmitter receptor was immunolabeled (Table 2). Within the superficial MDH, Fospositive neurons predominantly coexpressed AMPA glutamate receptors subunits GluA1 (95.8\%) and GluA2/3 (88.2\%), NMDA glutamate receptor subunits GluN1 (89.9\%) and GluN2A (41.4\%), and SP NK1 (64.1\%) receptors (Figure 3, Table 2). Other AMPA, NMDA, and kainate receptor subunits were not coexpressed to any great extent by Fos-positive neurons (Table 2). Within the deep MDH, Fos-positive neurons predominantly coexpressed GluA1 (91.7\%) and GluN1 (94.4\%) subunits and to a lesser extent GluA2/3 (25.0\%) and GluN2A (40.1\%) subunits and SP NK1 (40.8\%) receptors (Table 2). Within the paratrigeminal nucleus, Fos-positive neurons predominantly coexpressed GluA1 (93.1\%), GluA2/3 (75.0\%), GluN1 (95.5\%), and GluN2A (57.3\%) subunits and to a lesser extent SP NK1 (19.5\%) receptors (Table 2). 
TABLE 2: Percent colocalization of neurotransmitter receptor subunits with Fos-positive neurons. Percentages for each neurotransmitter receptor were determined by dividing the number of colocalized Fos-positive neurons by the total number of Fos-positive neurons present within each location.

\begin{tabular}{lccc}
\hline & Superficial MDH (laminae I and II) & Deep MDH (laminae III-V) & Paratrigeminal \\
\hline AMPA & & & $93.0 \pm 4.5$ \\
GluA1 & $95.8 \pm 1.0$ & $25.0 \pm 25.0$ & $75.0 \pm 9.0$ \\
GluA2/3 & $88.2 \pm 5.0$ & $0.0 \pm 0.0$ & $11.1 \pm 11.1$ \\
GluA4 & $3.0 \pm 3.0$ & & $0.0 \pm 0.0$ \\
Kainate & & $6.1 \pm 6.1$ & $12.9 \pm 4.8$ \\
GluK1/2/3 & $3.8 \pm 2.6$ & $4.2 \pm 2.8$ & $3.4 \pm 1.5$ \\
GluK4 & $2.9 \pm 1.4$ & $0.0 \pm 0.0$ & $95.5 \pm 2.4$ \\
GluK5 & $0.1 \pm 0.1$ & & $57.3 \pm 3.8$ \\
NMDA & & $94.4 \pm 2.4$ & $15.3 \pm 9.6$ \\
GluN1 & $89.9 \pm 2.6$ & $40.1 \pm 3.2$ & $0.0 \pm 0.0$ \\
GluN2A & $41.4 \pm 3.5$ & $16.2 \pm 7.3$ & $0.0 \pm 0.0$ \\
GluN2B & $7.6 \pm 3.0$ & $0.0 \pm 0.0$ & $7.9 \pm 3.7$ \\
GluN2C & $0.0 \pm 0.0$ & $0.0 \pm 0.0$ & $10.8 \pm 5.5$ \\
GluN2D & $0.0 \pm 0.0$ & & \\
GluN3B & $5.9 \pm 1.7$ & $40.8 \pm 2.9$ & \\
Substance P & & & \\
NK1 & $64.0 \pm 4.1$ & & \\
\hline
\end{tabular}

\section{Discussion}

Stimulation of the nasal mucosa of rats with ammonia vapors produces prolonged apnea, a significant decrease in $\mathrm{HR}$, and a significant increase in mean arterial pressure. As part of the central neuronal circuitry of this response, there is activation of neurons within the spinal trigeminal nucleus, specifically the MDH. The novel results from the present study indicate that during stimulation of the nasal mucosa of rats with ammonia vapors, many of these activated $\mathrm{MDH}$ neurons coexpress the AMPA glutamate receptor subunits GluA1 and GluA2/3 and the NMDA glutamate receptor subunits GluN1 and GluN2A, and a significant proportion coexpresses NK1, the receptor for SP.

The cardiorespiratory responses resulting from repetitive stimulation of the nasal mucosa with ammonia vapors has been described previously in both rats $[3,6]$ and muskrats [7]. Consistent with these results, we found that nasal stimulation produced apnea, significant bradycardia, and a significant increase in $\mathrm{BPa}$. The efferent aspects of this reflex response may be mediated by brainstem autonomic areas, including the nucleus tractus solitarius, the ventrolateral medulla, the A5 area, and the peribrachial complex [27]. The AEN innervates the nasal mucosa and is essential for the initiation of the afferent portion of this nasopharyngeal response [6]. The AEN projects primarily to the ipsilateral superficial portions of the ventral $\mathrm{MDH}$ and the paratrigeminal nuclei located within the ventral spinal trigeminal tract $[3,28]$. Neurons in these regions express Fos after either nasal stimulation $[6,8,29]$ or voluntary diving [5], and a similar pattern of Fos labeling was found within the $\mathrm{MDH}$ and paratrigeminal nucleus after nasal stimulation in the present study. Central projections of the AEN colocalize with $\mathrm{MDH}$ neurons activated by nasal stimulation, and the density of AEN terminal projections positively correlates with the rostral-caudal location of activated $\mathrm{MDH}$ neurons [3]. Presumably the afferent signals carried by the AEN activate these secondary neurons within the MDH [3]. These $\mathrm{MDH}$ neurons then likely project to other brainstem locations that are important in the production of the cardiorespiratory responses to nasal stimulation [28].

$c$-fos is an immediate early gene that encodes transcription factors which can participate in long-term alteration of cellular function [30]. As such, the immunological detection of Fos, the protein product of $c$-fos, has been used as a marker for neuronal activation within the CNS [30]. This technique appears to be particularly useful in identifying the afferent limb of a reflex circuit such as the diving response [7]. However the temporal pattern of Fos production and decay is dependent upon the brain region being investigated and type of stimulation used [31]. The production of Fos within secondary $\mathrm{MDH}$ neurons that results from intermittent and repetitive stimulation of the nasal mucosa has been previously described $[3,5-8]$. This protocol ( $2 \mathrm{hrs}$ of stimulation trials plus a $1 \mathrm{hr}$ wait) was chosen because Fos is produced within $\mathrm{MDH}$ neurons of animals receiving nasal stimulation but not within the $\mathrm{MDH}$ of unstimulated control animals $[6,7]$. For consistency and to extend the findings from these previous results, the present experiment used an identical stimulation protocol to ensure Fos production within $\mathrm{MDH}$ neurons. However, Fos can be expressed with minutes of neuronal activation [32] and receptor trafficking to the cell surface $[14,22]$ may have been altered by the nasal stimulation itself. It is therefore possible that between activation of the cardiovascular response by nasal stimulation and the termination of the experiment after a $1 \mathrm{hr}$ wait, there may have been an alteration in receptor distribution due to changes in receptor internalization or expression. Thus a potential limitation of our results may be that coexpression of Fos and receptors represents coexpression at the time of 

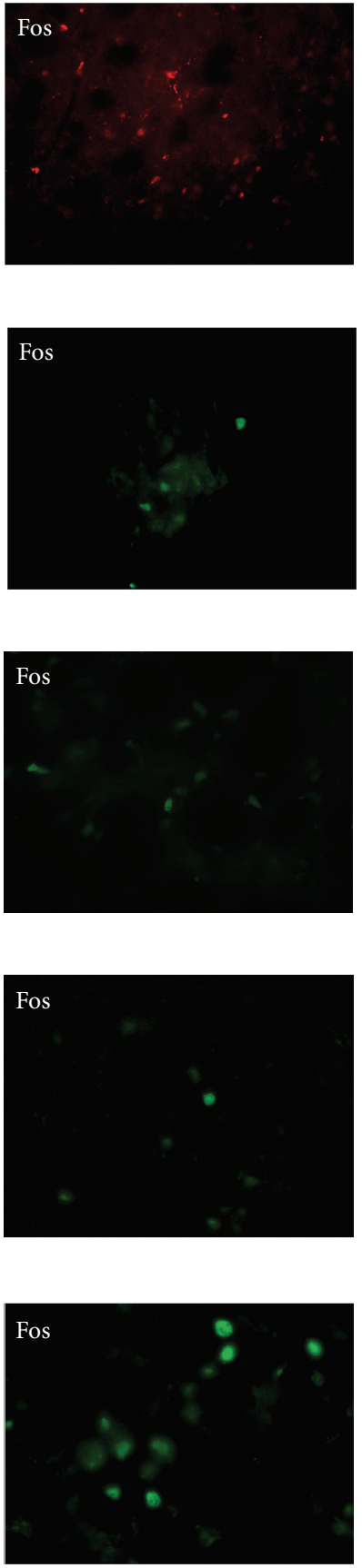

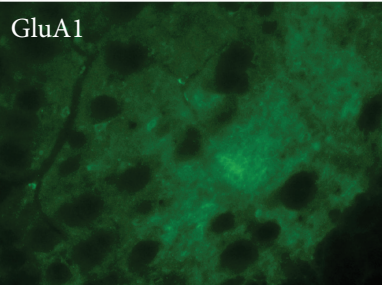

(a) Fos and GluA1

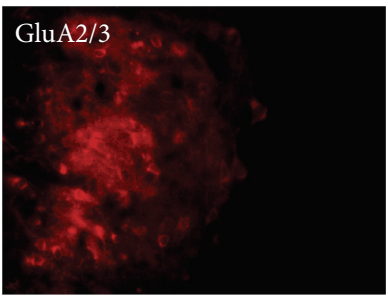

(b) Fos and GluA2/3

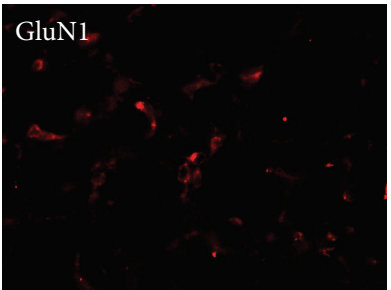

(c) Fos and GluN1

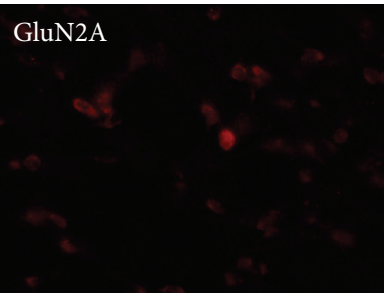

(d) Fos and GluN2A

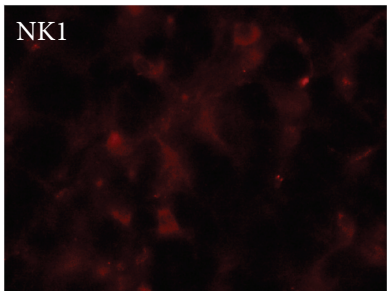

(e) Fos and NK1
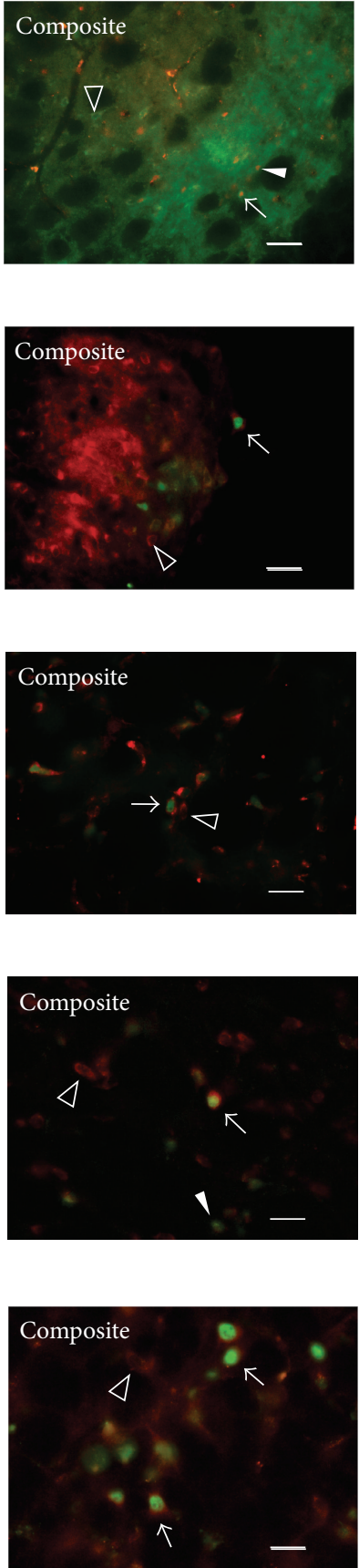

FIGURE 3: Fluorescent photomicrographic images showing individual Fos (left column), neurotransmitter subunit or receptor (middle column), and composite colocalized labeling (right column). Within the superficial MDH filled triangles indicate Fos-positive neurons, open arrowheads indicate Fos-negative but subunit or receptor positive neurons, and arrows indicate Fos-positive colocalized neurons. Scale bars: (a) $50 \mu \mathrm{m}$, (b)-(e) $25 \mu \mathrm{m}$.

euthanasia rather than coexpression at the time of actual nasal stimulation. While this may be a possibility, we feel that the receptor coexpression reported in these experiments is representative of what occurs during nasal stimulation and therefore helps to characterize the MDH neurons involved in mediating the cardiorespiratory changes induced after nasal stimulation.
Given the caveat just stated above, the results from the present experiments indicate that secondary neurons within the ventral portion of the $\mathrm{MDH}$ that are activated by nasal stimulation with ammonia vapors express AMPA, NMDA and NK1 receptors, but not kainate receptors (Figure 3, Table 2). Secondary MDH neurons have previously been found to express both AMPA, and NMDA glutamate 
receptors $[33,34]$. Because the AEN innervates the nasal passages, sends central projections to the $\mathrm{MDH}$ location, and induces activation of $\mathrm{MDH}$ neurons [3], and since these $\mathrm{MDH}$ neurons express AMPA, NMDA, and NK1 receptors (present study), our results suggest that the AEN releases glutamate as a neurotransmitter and SP as a cotransmitter/neuromodulator after stimulation of the nasal passages. Presumably glutamate and/or SP released by the AEN activate these secondary $\mathrm{MDH}$ neurons and induce them to produce Fos through activation of multiple glutamatergic and SP receptors.

Within the superficial layers (laminae I and II) of the $\mathrm{MDH}$ quantitative autoradiography indicates a high density of AMPA receptors [35]. With regards to AMPA receptor subunits, GluA1 is expressed moderately, GluA2/3 is intensely expressed, and GluA4 is not expressed [36]. In the present study, over $90 \%$ of $\mathrm{MDH}$ neurons activated by nasal stimulation (both within the superficial and deep MDH laminae, as well as in the adjacent paratrigeminal region) coexpress the glutamate receptor subunit GluA1 (Figure 3, Table 2). Additionally, GluA2/3 glutamate receptor subunits are coexpressed by over $75 \%$ of neurons within the superficial $\mathrm{MDH}$ and paratrigeminal region. Since GluA1, GluA2, and GluA3 are all subunits that form AMPA receptors, this suggests that AMPA receptors are present on these secondary $\mathrm{MDH}$ neurons. The involvement of AMPA receptors in the physiological responses to nasal stimulation has been suggested previously, as infusion of DNQX, an antagonist of non-NMDA glutamate receptors, into the spinal trigeminal nucleus reversibly eliminates the cardiorespiratory responses to nasal stimulation [37]. Since AMPA receptors mediate fast synaptic neurotransmission $[10,12,14]$, this glutamatergic mechanism may mediate the fast cardiorespiratory reflex responses that result from nasal stimulation (Figure 2). Electrophysiological evidence indicates that neurons within lamina I of the $\mathrm{MDH}$ produce AMPA receptor-mediated excitatory postsynaptic currents after electrical stimulation of the spinal trigeminal tract [38].

There may also be a slower neurosynaptic mechanism within the $\mathrm{MDH}$ involving glutamate release by the central terminations of the AEN, as activated $\mathrm{MDH}$ neurons coexpress the NMDA receptor subunits GluN1 and GluN2A (Table 2). GluN1 receptor subunits are strongly expressed in the $\mathrm{MDH}[39,40]$. Previous results have indicated that NMDA receptors may be involved in producing the cardiorespiratory responses elicited by nasal stimulation. Kynurenic acid, an antagonist of the glycine binding site located on the GluN1 subunit [13, 41], abolishes the cardiorespiratory responses to nasal stimulation when infused into the $\mathrm{MDH}$ [9]. AP7, an NMDA receptor antagonist, reversibly eliminates the cardiorespiratory responses to nasal stimulation when injected into the spinal trigeminal nucleus [37]. Also, stimulation of the nasal mucosa with saline produces a significant increase in Fos-like immunoreactivity within the $\mathrm{MDH}$, and $53 \%$ of these Fos-positive neurons are also immunoreactive for the GluN1 receptor subunit [8]. Finally, electrical stimulation of the spinal trigeminal tract produces NMDA receptor-mediated excitatory postsynaptic currents within lamina I MDH neurons [38]. These studies and the present results all suggest that glutamatergic neurotransmission in the $\mathrm{MDH}$ after nasal stimulation is at least partially dependent on NMDA receptors.

NK1 receptors were coexpressed by approximately $50 \%$ of activated $\mathrm{MDH}$ neurons within the superficial and deep $\mathrm{MDH}$ and by $20 \%$ of activated MDH neurons within the paratrigeminal nucleus. However it is possible that NK1 receptor internalization after activation by SP $[19,22]$ may have caused an underrepresentation of the number of activated neurons that coexpressed NK1 receptors. Within the dorsal horn glutamate and SP have been shown to coexist in primary afferent C-fibers [42] and are coreleased by primary afferent neurons involved in mediating nociception [23, 43]. NMDA receptors are frequently located in the postsynaptic targets of SP terminals and may play a role in the modulation of SP containing neurons [44]. Additionally, glutamate activated conductance in rat spinal dorsal horn neurons is enhanced by SP [23]. Many lines of evidence indicate that SP and NK1 receptors play an important role in nociception [17-20, 24], and pain associated with peripheral tissue or nerve injury involves NMDA receptor activation [45]. Because there is activation of MDH neurons expressing both NMDA and NK1 receptors, the present results suggest that at least part of the signal originating from the nasal passages that initiates the observed cardiorespiratory responses is nociceptive in nature. In support of this contention, approximately $65 \%$ of the muskrats AEN is composed of unmyelinated C-fibers, and $72 \%$ of the AEN myelinated fibers are of the A- $\delta$ small diameter type [46]. Because nociceptive pathways utilize small diameter fibers [47], the fact that the AEN contains roughly $90 \%$ small diameter fibers [46] suggests that the AEN may be involved in nociceptive signaling. Additionally, the superficial region of the dorsal horn receives direct input from myelinated and unmyelinated nociceptors [47] and was where $69.9 \%$ of the activated (Fos-positive) $\mathrm{MDH}$ neurons were located in the present study.

\section{Conclusion}

The present study has identified neurotransmitter receptors present on $\mathrm{MDH}$ neurons that are activated during nasal stimulation. Glutamate receptors include both AMPA and NMDA, but not kainate, subtypes. Additionally, many of the activated $\mathrm{MDH}$ neurons also coexpressed NK1 receptors. It is therefore likely that the terminal fibers of the anterior ethmoidal nerve that synapse with $\mathrm{MDH}$ neurons release glutamate and SP to activate these $\mathrm{MDH}$ neurons during nasal stimulation. The involvement of AMPA and NMDA receptors may mediate fast and slow neurotransmission, respectively, while NK1 receptors may indicate activation of a nociceptive pathway.

\section{Highlights}

(1) Nasal stimulation produces apnea, bradycardia, and increased arterial blood pressure.

(2) Neurons within trigeminal medullary dorsal horn are activated by nasal stimulation. 
(3) Activated trigeminal medullary dorsal horn neurons express AMPA, NMDA, and NK1 receptors.

\section{Abbreviations}

AEN: Anterior ethmoidal nerve

AMPA: $\alpha$-Amino-3-hydroxy-5-methyl-4isoxazole-propionate glutamate receptors

BPa: Arterial blood pressure

BSA: Bovine serum albumin

HR: Heart rate

MDH: Medullary dorsal horn

NK1: Neurokinin substance P receptor

NMDA: N-Methyl-D-aspartate glutamate receptors

PBS: $\quad$ Phosphate buffered saline

SE: $\quad$ Standard error

SDH: Spinal dorsal horn

SP: $\quad$ Substance P.

\section{Acknowledgments}

Research was sponsored by Midwestern University Office of Research and Sponsored Programs (PFM), College of Health Sciences Master's in Biomedical Sciences Program (D. J. Westerhaus, J. F. Peevey), and Chicago College of Osteopathic Medicine Summer Research Fellowship Program (T. A. Vizinas, M. A. Lach, and P. Czarnocki).

\section{References}

[1] P. J. Butler and D. R. Jones, "Physiology of diving of birds and mammals," Physiological Reviews, vol. 77, no. 3, pp. 837-899, 1997.

[2] P. F. McCulloch, "Animal models for investigating the central control of the mammalian diving response," Frontiers in Physiology, vol. 3, pp. 1-16, 2012.

[3] M. P. Hollandsworth, K. M. DiNovo, and P. F. McCulloch, "Unmyelinated fibers of the anterior ethmoidal nerve in the rat co-localize with neurons in the medullary dorsal horn and ventrolateral medulla activated by nasal stimulation," Brain Research, vol. 1298, pp. 131-144, 2009.

[4] S. Gobel, S. Hockfield, and M. Ruda, "Anatomical similarities between medullary and spinal dorsal horns," in Oral-Facial Sensory and Motor Functions, Y. Kawamura and R. Dubner, Eds., pp. 211-223, Quintessence, Tokyo, Japan, 1988.

[5] P. F. McCulloch, "Activation of the trigeminal medullary dorsal horn during voluntary diving in rats," Brain Research, vol. 1051, no. 1-2, pp. 194-198, 2005.

[6] E. J. Rybka and P. F. McCulloch, "The anterior ethmoidal nerve is necessary for the initiation of the nasopharyngeal response in the rat," Brain Research, vol. 1075, no. 1, pp. 122-132, 2006.

[7] P. F. McCulloch and W. M. Panneton, "Fos immunohistochemical determination of brainstem neuronal activation in the muskrat after nasal stimulation," Neuroscience, vol. 78, no. 3, pp. 913-925, 1997.

[8] M. Dutschmann, A. Guthmann, and H. Herbert, "NMDA receptor subunit NR1-immunoreactivity in the rat pons and brainstem and colocalization with Fos induced by nasal stimulation," Brain Research, vol. 809, no. 2, pp. 221-230, 1998.
[9] W. M. Panneton and P. Yavari, "A medullary dorsal horn relay for the cardiorespiratory responses evoked by stimulation of the nasal mucosa in the muskrat Ondatra zibethicus: evidence for excitatory amino acid transmission," Brain Research, vol. 691, no. 1-2, pp. 37-45, 1995.

[10] S. Ozawa, H. Kamiya, and K. Tsuzuki, "Glutamate receptors in the mammalian central nervous system," Progress in Neurobiology, vol. 54, no. 5, pp. 581-618, 1998.

[11] B. S. Meldrum, "Glutamate as a neurotransmitter in the brain: review of physiology and pathology," Journal of Nutrition, vol. 130, supplement, no. 4, pp. 1007S-1015S, 2000.

[12] C. G. Parsons, W. Danysz, and W. Zieglgänsberger, "Excitatory amino acid neurotransmission," Handbook of Experimental Pharmacology, no. 169, pp. 249-303, 2005.

[13] R. Dingledine, K. Borges, D. Bowie, and S. F. Traynelis, "The glutamate receptor ion channels," Pharmacological Reviews, vol. 51, no. 1, pp. 7-61, 1999.

[14] S. F. Traynelis, L. P. Wollmuth, C. J. McBain et al., "Glutamate receptor ion channels: structure, regulation, and function," Pharmacological Reviews, vol. 62, no. 3, pp. 405-496, 2010.

[15] S. Bassani, P. Valnegri, F. Beretta, and M. Passafaro, "The GLUR2 subunit of AMPA receptors: synaptic role," Neuroscience, vol. 158, no. 1, pp. 55-61, 2009.

[16] D. Bowie, "Ionotropic glutamate receptors \& CNS disorders," CNS and Neurological Disorders, vol. 7, no. 2, pp. 129-143, 2008.

[17] R. G. Hill and K. R. Oliver, "Neuropeptide and kinin antagonists," Handbook of Experimental Pharmacology, no. 177, pp. 181216, 2007.

[18] P. Datar, S. Srivastava, E. Coutinho, and G. Govil, "Substance P: structure, function, and therapeutics," Current Topics in Medicinal Chemistry, vol. 4, no. 1, pp. 75-103, 2004.

[19] P. W. Mantyh, "Neurobiology of substance P and the NK1 receptor," Journal of Clinical Psychiatry, vol. 63, no. 11, pp. 6-10, 2002.

[20] T. Hökfelt, B. Pernow, and J. Wahren, "Substance P: a pioneer amongst neuropeptides," Journal of Internal Medicine, vol. 249, no. 1, pp. 27-40, 2001.

[21] A. Saria, "The tachykinin NK1 receptor in the brain: pharmacology and putative functions," European Journal of Pharmacology, vol. 375, no. 1-3, pp. 51-60, 1999.

[22] L. Quartara and C. A. Maggi, "The tachykinin NK1 receptor. Part I: ligands and mechanisms of cellular activation," Neuropeptides, vol. 31, no. 6, pp. 537-563, 1997.

[23] M. Randic, M. Kolaj, L. Kojic, R. Cerne, G. Cheng, and R. A. Wang, "Interaction of neuropeptides and excitatory amino acids in the rat superficial spinal dorsal horn," Progress in Brain Research, vol. 104, pp. 225-253, 1994.

[24] L. Quartara and C. A. Maggi, “The tachykinin receptor. Part II: distribution and pathophysiological roles," Neuropeptides, vol. 32, no. 1, pp. 1-49, 1998.

[25] E. Bullitt, "Expression of c-fos-like protein as a marker for neuronal activity following noxious stimulation in the rat," Journal of Comparative Neurology, vol. 296, no. 4, pp. 517-530, 1990.

[26] G. Paxinos and C. Watson, The Rat Brain in Stereotaxic Coordinates, Academic Press, New York, NY, USA, 4th edition, 1998.

[27] W. M. Panneton, P. F. McCulloch, and W. Sun, "Trigeminoautonomic connections in the muskrat: the neural substrate for the diving response," Brain Research, vol. 874, no. 1, pp. 48-65, 2000.

[28] W. M. Panneton, Q. Gan, and R. Juric, "Brainstem projections from recipient zones of the anterior ethmoidal nerve in the 
medullary dorsal horn," Neuroscience, vol. 141, no. 2, pp. 889906, 2006.

[29] M. Dutschmann and H. Herbert, "Fos expression in the rat parabrachial and Kolliker-Fuse nuclei after electrical stimulation of the trigeminal ethmoidal nerve and water stimulation of the nasal mucosa," Experimental Brain Research, vol. 117, no. 1, pp. 97-110, 1997.

[30] J. I. Morgan and T. Curran, "Stimulus-transcription coupling in the nervous system: involvement of the inducible protooncogenes fos and jun," Annual Review of Neuroscience, vol. 14, pp. 421-451, 1991.

[31] M. Dragunow and R. Faull, "The use of c-fos as a metabolic marker in neuronal pathway tracing," Journal of Neuroscience Methods, vol. 29, no. 3, pp. 261-265, 1989.

[32] J. I. Morgan, D. R. Cohen, J. L. Hempstead, and T. Curran, "Mapping patterns of c-fos expression in the central nervous system after seizure," Science, vol. 237, no. 4811, pp. 192-196, 1987.

[33] S.-K. Leong, H.-P. Liu, and J.-F. Yeo, "Nitric oxide synthase and glutamate receptor immunoreactivity in the rat spinal trigeminal neurons expressing Fos protein after formalin injection," Brain Research, vol. 855, no. 1, pp. 107-115, 2000.

[34] F. Florenzano and B. De Luca, "Nociceptive stimulation induces glutamate receptor down-regulation in the trigeminal nucleus," Neuroscience, vol. 90, no. 1, pp. 201-207, 1999.

[35] S. J. Tallaksen-Greene, A. B. Young, J. B. Penney, and A. J. Beitz, "Excitatory amino acid binding sites in the trigeminal principal sensory and spinal trigeminal nuclei of the rat," Neuroscience Letters, vol. 141, no. 1, pp. 79-83, 1992.

[36] F. R. Tang, J. F. Yeo, and S. K. Leong, "Qualitative light and electron microscope study of glutamate receptors in the caudal spinal trigeminal nucleus of the rat," Journal of Dental Research, vol. 80, no. 8, pp. 1736-1741, 2001.

[37] P. F. McCulloch, I. A. Paterson, and N. H. West, "An intact glutamatergic trigeminal pathway is essential for the cardiac response to simulated diving," The American Journal of Physiology, vol. 269, no. 3, pp. R669-R677, 1995.

[38] M. Sedlacek, M. Horak, and L. VyklickÝ Jr., "Morphology and physiology of lamina I neurons of the caudal part of the trigeminal nucleus," Neuroscience, vol. 147, no. 2, pp. 325-333, 2007.

[39] A. Guthmann and H. Herbert, "Expression of N-methyl-Daspartate receptor subunits in the rat parabrachial and KollikerFuse nuclei and in selected pontomedullary brainstem nuclei," Journal of Comparative Neurology, vol. 415, no. 4, pp. 501-517, 1999.

[40] R. S. Petralia, N. Yokotani, and R. J. Wenthold, "Light and electron microscope distribution of the NMDA receptor subunit NMDAR1 in the rat nervous system using a selective antipeptide antibody," Journal of Neuroscience, vol. 14, no. 2, pp. 667-696, 1994.

[41] J. C. Watkins and D. E. Jane, “The glutamate story," British Journal of Pharmacology, vol. 147, supplement 1, pp. S100-S108, 2006.

[42] S. De Biasi and A. Rustioni, "Glutamate and substance P coexist in primary afferent terminals in the superficial laminae of spinal cord," Proceedings of the National Academy of Sciences of the United States of America, vol. 85, no. 20, pp. 7820-7824, 1988.

[43] G. Battaglia and A. Rustioni, "Coexistence of glutamate and substance $\mathrm{P}$ in dorsal root ganglion neurons of the rat and monkey," Journal of Comparative Neurology, vol. 277, no. 2, pp. 302-312, 1988.
[44] S. A. Aicher, S. Sharma, P. Y. Cheng, and V. M. Pickel, "The $\mathrm{N}$-methyl-D-aspartate (NMDA) receptor is postsynaptic to substance P-containing axon terminals in the rat superficial dorsal horn," Brain Research, vol. 772, no. 1-2, pp. 71-81, 1997.

[45] A. B. Petrenko, T. Yamakura, H. Baba, and K. Shimoji, “The role of N-methyl-D-aspartate (NMDA) receptors in pain: a review," Anesthesia and Analgesia, vol. 97, no. 4, pp. 1108-1116, 2003.

[46] P. F. McCulloch, K. M. Faber, and W. M. Panneton, "Electrical stimulation of the anterior ethmoidal nerve produces the diving response," Brain Research, vol. 830, no. 1, pp. 24-31, 1999.

[47] E. R. Perl, "Ideas about pain, a historical view," Nature Reviews Neuroscience, vol. 8, no. 1, pp. 71-80, 2007. 

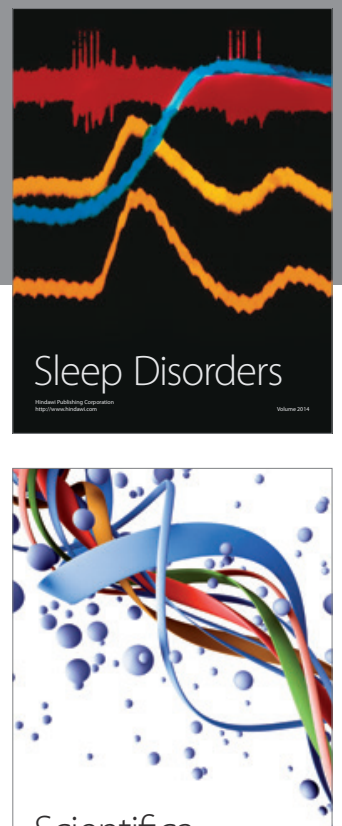

Scientifica
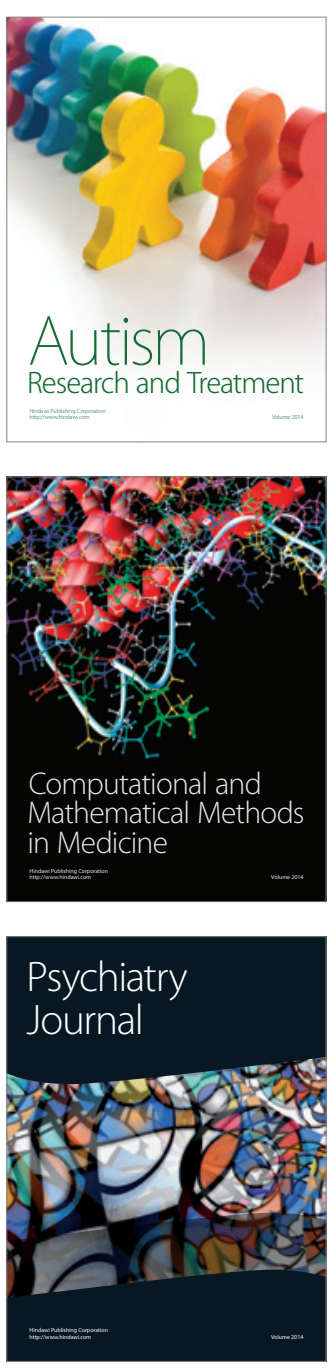
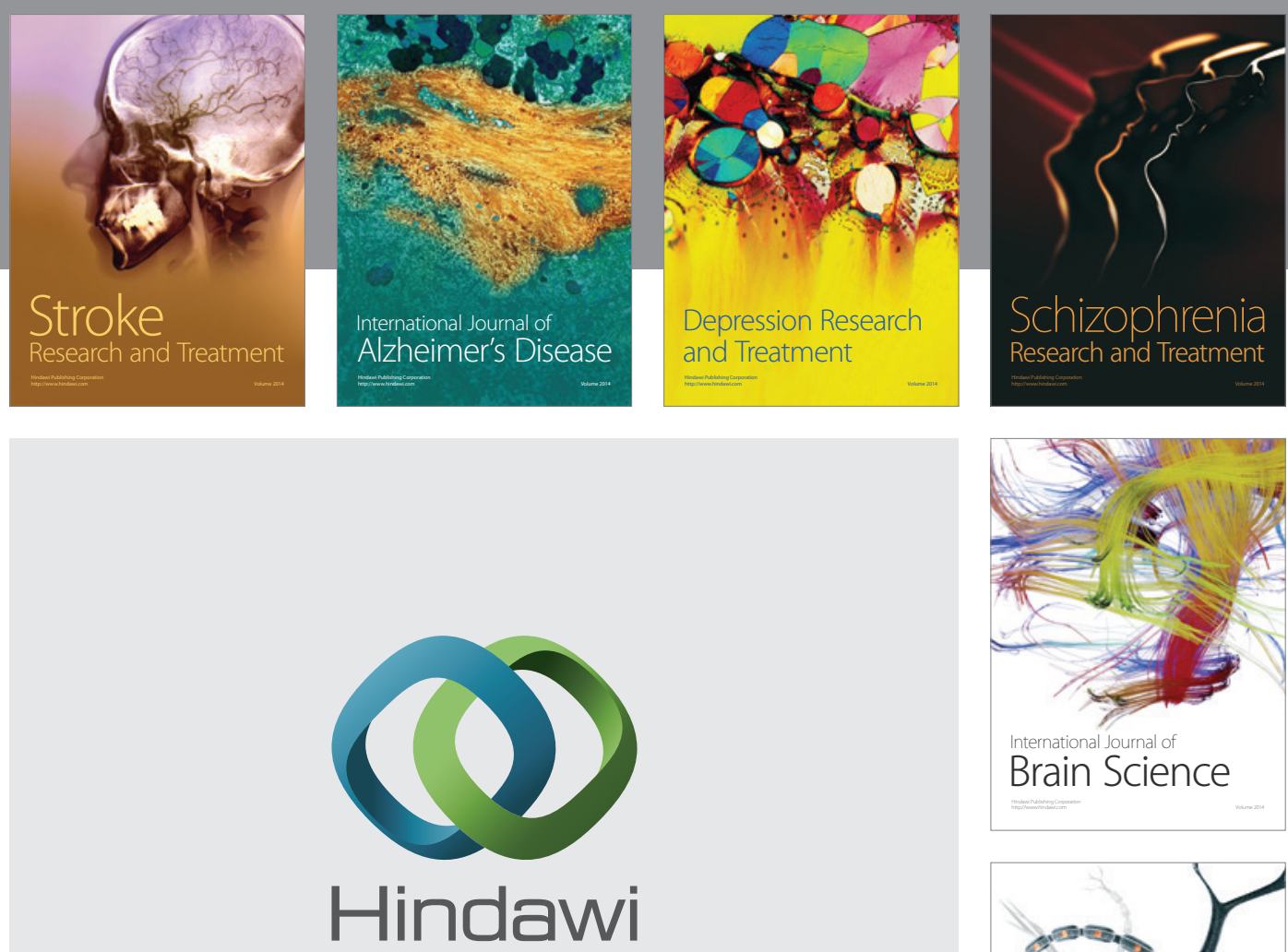

Submit your manuscripts at

http://www.hindawi.com
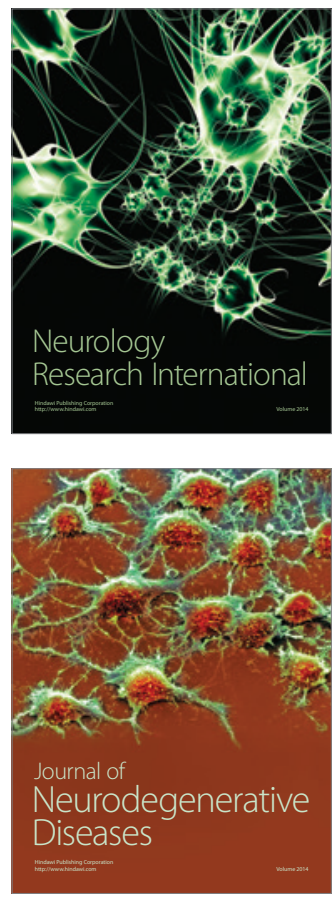

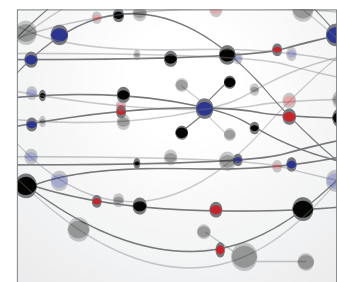

The Scientific World Journal
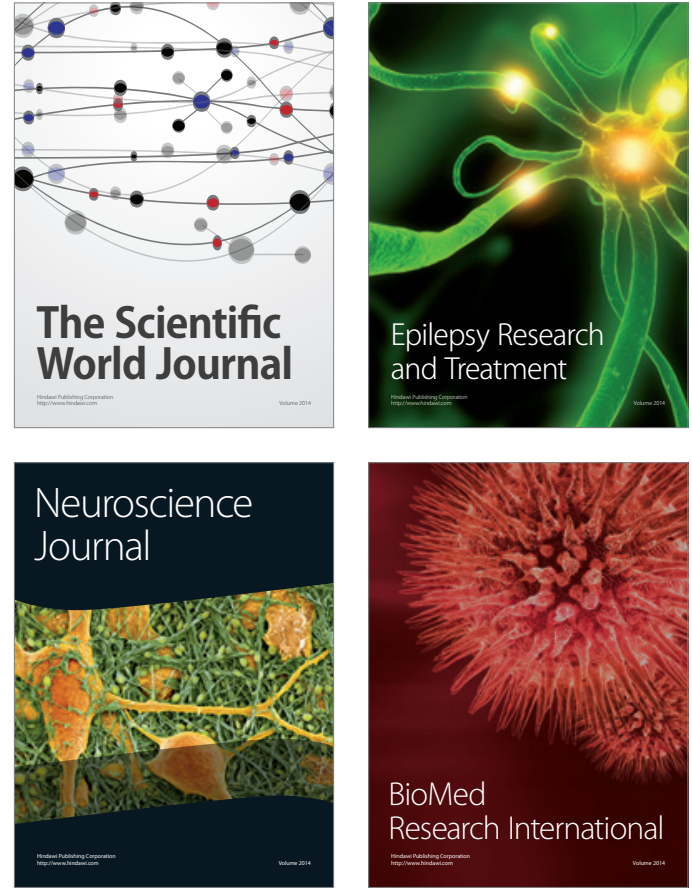

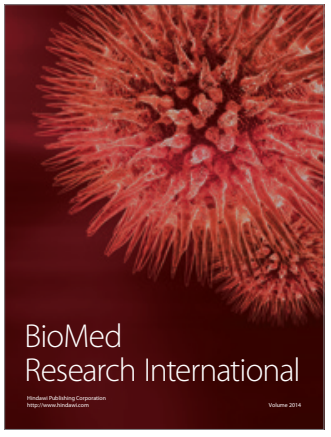

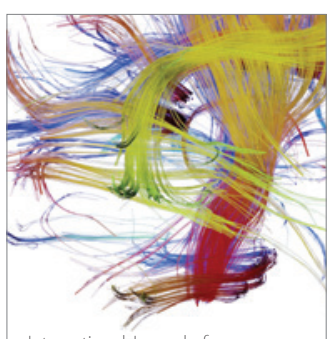

Brain Science

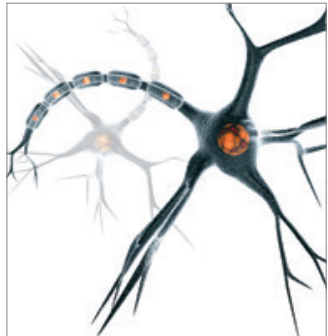

Neural Plasticity
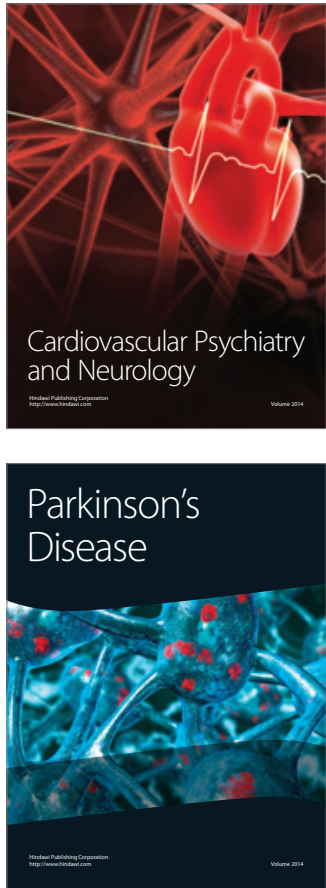